\title{
On Efforts in Teams with Stereotypes*
}

\author{
Shiva Sikdar ${ }^{\dagger}$
}

\begin{abstract}
Diversity in the workplace implies a balance in positions held by different social groups in organizations. We analyze the effect of negative stereotypes about the abilities of individuals from disadvantaged backgrounds on efforts and outcomes in teams. A project's success depends on the abilities and efforts of agents from different backgrounds. Under simultaneous effort contribution, the stereotype lowers efforts of all agents and the project's success chance. When the principal assigns the disadvantaged/stereotyped agent as leader in effort contribution, the effect of the stereotype is mitigated and the project's success chance is the highest; this also maximizes the principal's expected payoff. Although the principal offers symmetric incentives, the stereotyped agent often exerts higher effort.
\end{abstract}

JEL Classification: J71, D2.

Key Words: Stereotype, team production, sequential effort contribution, peer effect.

*I am thankful to the Editor, Roberto Serrano, and an anonymous referee for their comments and suggestions, which significantly improved the paper. I also thank Parimal Bag, Harvey Lapan and Bibhas Saha for helpful comments. The usual disclaimer applies.

${ }^{\dagger}$ Keele Management School, Darwin Building, Keele ST5 5BG, UK; E-mail: shivasikdar@gmail.com 


\section{Introduction}

Most organizations seek to accomplish diversity in the workplace by achieving a balance in positions held by individuals from different social groups. However, often, there are negative stereotypes about the abilities of individuals from disadvantaged groups - say, due to historical reasons or because it might be considered that affirmative action makes it easier for candidates from such social backgrounds to get a position. Coate and Loury (1993) show that, in a model of statistical discrimination, negative stereotypes can persist due to affirmative action; see, also, Hoff and Stiglitz (2010). See Jones (2002) for the psychological aspect of stereotyping. Further, almost all Fortune 1000 companies use team projects, but $90 \%$ of teams result in failures (Shaw and Schneier, 1995). ${ }^{1}$ Given the importance of diversity and team production in organizations, we analyze the impact of stereotypes in such settings and how production can be structured to reduce their impact.

Previous work has shown the positive impact of biased self-perception on performance. ${ }^{2}$ Gervais and Goldstein (2007) analyze the effect of having an overconfident team-member (who believes her productivity is higher than it actually is) when there are peer effects (complementarities). Overconfidence can improve outcomes for the team and the principal as this agent exerts higher effort than she would otherwise. In contrast, we focus on the impact of negative stereotypes about other team-members' abilities based on social backgrounds..$^{3,4}$

In our model, two agents from different backgrounds, advantaged and disadvantaged, are employed to work in a team. The project's success depends on the agents' efforts and abilities. The advantaged agent believes a negative stereotype about the disadvantaged agent - that the latter's ability is lower than it is actually. Our results highlight the negative impact of such stereotype in team settings with complementarity (peer effects). Under simultaneous effort contribution, the advantaged agent exerts low effort because she underestimates the disadvantaged agent's effort (hence, the marginal productivity of her own effort). The stereotyped agent internalizes this bias which, in turn, reduces the marginal productivity of her effort (due to the advantaged agent's low effort) and also exerts low effort.

Making effort contribution sequential increases efforts relative to simultaneous efforts - with the stereotyped agent as leader, efforts (and hence, the project's success chance) are the highest. ${ }^{5}$ When the disadvantaged agent leads, the advantaged agent observes the former's effort and does not have to rely on her beliefs about this. Hence, the negative impact of the stereotype is

\footnotetext{
${ }^{1}$ The classic paper dealing with incentives in teams is Holmström (1982); see, also, Lazear and Shaw (2007).

${ }^{2}$ Compte and Postlewaite (2004) show that self-perception biases can be optimal when confidence influences an individual's performance.

${ }^{3}$ Chaudhuri and Sethi (2008) analyze the effect of stereotypes on human capital investment in a model of statistical discrimination with peer effects. Empirical evidence on the negative impact of affirmative action through peer effects in education have been provided in Kochar (2010) and Sekhri (2012).

${ }^{4}$ Although one might say that team-members may not be aware of each others social identity, in India, for instance, surnames can help identify one's religion/caste; see Banerjee, et al. (2009) and Bertrand and Mullainathan (2004). Further, it is natural for individuals to have some information about the background of co-workers in a team; in case of gender, color, etc., there is no effective way to hide these characteristics. Even if background were not fully visible, one can form beliefs about a co-worker's background.

${ }^{5}$ See Hermalin (1998) for a signaling model of leadership. Andreoni (2006) shows how 'leadership giving' can increase charitable contribution when it provides a signal of the charity's quality.
} 
mitigated, resulting in the highest expected payoff for the principal. It is always optimal for the principal to offer agents symmetric incentives. As the stereotype becomes more pronounced, the disadvantaged agent's effort increases relative to the advantaged agent. This is because the former (correctly) perceives a higher marginal productivity of her effort than the latter who believes the stereotype.

These results are also related to the sequential contribution literature on public goods provision. Varian (1994) shows that, under complete information and substitutability, sequential contribution worsens the free rider problem and total contribution is generally lower than that under simultaneous contributions. One might expect the stereotype to further exacerbate the usual free riding problem. However, in team settings with stereotypes and peer effects, the outcomes are counter to Varian (1994). ${ }^{6}$

\section{The Model}

A risk-neutral principal employs two risk-neutral agents to work in a team for a joint project. Agents belong to two different social backgrounds, $i \in\{A, B\} ; A$ and $B$ denote advantaged and disadvantaged groups, respectively. We denote both the group and agent by $i \in\{A, B\}$.

The probability of the project's success is given by a standard Cobb-Douglas function:

$$
P=P\left(\eta_{A}, \eta_{B}, e_{A}, e_{B}\right)=\eta_{A}^{\alpha} e_{A}^{1-\alpha} \eta_{B}^{\alpha} e_{B}^{1-\alpha}, \quad \alpha \in(0,1)
$$

where $\eta_{i}$ and $e_{i}$ are the ability and effort of agent $i$, respectively, $i \in\{A, B\}$. Hence, there are peer effects due to the complementarity of efforts of the agents.

If the project succeeds, it generates a payoff of $V$ for the principal, who is unable to observe efforts, so cannot condition agent payoffs directly on efforts but only on outcomes. Agent $i$ is paid $v_{i}$ when the project succeeds and zero otherwise. We normalize the agents' outside option to zero. The cost of effort is $C\left(e_{i}\right)=\frac{1}{2} e_{i}^{2}$. Hence, agent $i$ 's utility is:

$$
U_{i}= \begin{cases}v_{i}-\frac{1}{2} e_{i}^{2}, & \text { if the project succeeds } \\ -\frac{1}{2} e_{i}^{2}, & \text { if the project fails. }\end{cases}
$$

The principal's net payoff is:

$$
W= \begin{cases}V-v_{A}-v_{B}, & \text { if the project succeeds } \\ 0, & \text { if the project fails. }\end{cases}
$$

Agents know their own ability, i.e., there is no self-perception bias. Their belief about the other agent's ability may not be accurate. Agent $j$ 's perception/belief about $i$ 's ability is $\tilde{\eta}_{i}$, $i, j \in\{A, B\}, i \neq j$. Agent $B$ 's belief about $A$ 's ability is accurate, i.e., $\tilde{\eta}_{A}=\eta_{A}$. However,

Assumption 1 (Stereotype). Agent $A$ believes a negative stereotype about B: specifically, $A$ believes that $B$ 's ability is lower than it actually is, i.e., $\tilde{\eta}_{B}=\eta_{B}-b<\eta_{B}, 0<b<\eta_{B}$.

\footnotetext{
${ }^{6}$ Bag and Roy (2011) show that, with incomplete information about private valuations for a public good, sequential contributions can result in higher total contribution than under simultaneous contributions.
} 
We also assume that $A$ knows that $B$ believes $\tilde{\eta}_{A}=\eta_{A}$ and $B$ knows that $A$ believes $\tilde{\eta}_{B}=\eta_{B}-b$. The model is kept simple to focus on our key issue - the impact of stereotypes about an agent's ability (an unobservable characteristic) based on her social background (an observable characteristic). ${ }^{7}$ Further, although the principal knows both agents' abilities, ${ }^{8}$ she cannot credibly communicate this to either agent.

\section{Benchmark: Simultaneous Effort Contributions}

When agents exert effort simultaneously, given $\left\{v_{A}, v_{B}\right\}$, the overall efforts are determined by the Nash equilibrium of a simultaneous-move unobservable effort contribution game.

Given $\left\{v_{A}, v_{B}\right\}$, agent $A$ maximizes her expected utility

$$
\max _{e_{A} \geq 0} \mathbb{E} U_{A}=v_{A} \underbrace{\left[\eta_{A}^{\alpha}\left(\eta_{B}-b\right)^{\alpha} e_{A}^{1-\alpha} e_{B}^{1-\alpha}\right]}_{\mathbb{E}_{A}(P)}-\frac{1}{2} e_{A}^{2},
$$

where $\mathbb{E}_{A}(P)$ denotes $A$ 's belief about the project's success probability, which depends on her beliefs about $B$ 's ability (and effort). The stereotype implies that this belief is biased downward since $b>0$. The first-order condition gives $A$ 's best-response function: ${ }^{9}$

$$
e_{A}=\left[(1-\alpha) v_{A} \eta_{A}^{\alpha}\left(\eta_{B}-b\right)^{\alpha}\right]^{\frac{1}{1+\alpha}} \hat{e}_{B}^{\frac{1-\alpha}{1+\alpha}}
$$

where $\hat{e}_{B}$ is $A$ 's belief about $B$ 's effort choice.

Agent $B$ 's problem is (given $\left\{v_{A}, v_{B}\right\}$ )

$$
\max _{e_{B} \geq 0} \mathbb{E} U_{B}=v_{B} \underbrace{\left[\eta_{A}^{\alpha} \eta_{B}^{\alpha} e_{A}^{1-\alpha} e_{B}^{1-\alpha}\right]}_{\mathbb{E}_{B}(P)}-\frac{1}{2} e_{B}^{2},
$$

where $\mathbb{E}_{B}(P)$ denotes $B$ 's belief about the project's success probability. The first-order condition gives $B$ 's best-response function (where $\hat{e}_{A}$ denotes $B$ 's belief about $A$ 's effort choice):

$$
e_{B}=\left[(1-\alpha) v_{B} \eta_{A}^{\alpha} \eta_{B}^{\alpha}\right]^{\frac{1}{1+\alpha}} \hat{e}_{A}^{\frac{1-\alpha}{1+\alpha}}
$$

Complementarity of efforts (peer effects) implies each agent's effort is increasing in the other agent's effort: $\frac{\partial e_{A}}{\partial \hat{e}_{B}}>0$ and $\frac{\partial e_{B}}{\partial \hat{e}_{A}}>0$. Agent $A$ underestimates $B$ 's effort due to the stereotype, i.e., $\hat{e}_{B}=\left[(1-\alpha) v_{B} \eta_{A}^{\alpha}\left(\eta_{B}-b\right)^{\alpha}\right]^{\frac{1}{1+\alpha}} e_{A}^{\frac{1-\alpha}{1+\alpha}}$.

Solving the best-response functions simultaneously, taking into account the stereotype, we

\footnotetext{
${ }^{7}$ Suppose, in contrast to our simplifying assumption, neither agent knows the other agent's ability. Assume that there are two ability types, $\eta \in\left\{\eta_{h}, \eta_{\ell}\right\}, \eta_{h}>\eta_{\ell}$; agent $i$ 's subjective belief about $j$ being of high ability, $\eta_{h}$, is $\hat{p}_{j}$. If $A$ assigns a low value to $\hat{p}_{B}$ based on $B$ 's social background, our main results would apply.

${ }^{8}$ See Bag, et al. (2015) for effects of biased employer beliefs on hiring decisions under job market signaling.

${ }^{9}$ Details of the derivation of efforts and verification of the second-order conditions for the agents' and principal's problems are available as Supplementary Appendix.
} 
have the equilibrium efforts (with the superscript $S$ denoting simultaneous efforts):

$e_{A}^{S}=(1-\alpha)^{\frac{1}{2 \alpha}} v_{A}^{\frac{1+\alpha}{4 \alpha}} v_{B}^{\frac{1-\alpha}{4 \alpha}} \eta_{A}^{\frac{1}{2}}\left(\eta_{B}-b\right)^{\frac{1}{2}}$, and $e_{B}^{S}=(1-\alpha)^{\frac{1}{2 \alpha}} v_{A}^{\frac{1-\alpha}{4 \alpha}} v_{B}^{\frac{1+\alpha}{4 \alpha}} \eta_{A}^{\frac{1}{2}} \eta_{B}^{\frac{\alpha}{1+\alpha}}\left(\eta_{B}-b\right)^{\frac{1-\alpha}{2(1+\alpha)}}$.

It is straightforward to see that $\frac{\partial e_{i}^{S}}{\partial b}<0, i \in\{A, B\}$; hence, as $b \uparrow$, the effort of both agents falls. As the stereotype lowers both agents' efforts, it also lowers the project's success chance.

The probability of the project's success is (using (2)):

$$
P^{S}=\eta_{A}^{\alpha} \eta_{B}^{\alpha}\left(e_{A}^{S}\right)^{1-\alpha}\left(e_{B}^{S}\right)^{1-\alpha}=\underbrace{(1-\alpha)^{\frac{1-\alpha}{\alpha}} \eta_{A} \eta_{B}^{\frac{2 \alpha}{1+\alpha}}\left(\eta_{B}-b\right)^{\frac{1-\alpha}{1+\alpha}}}_{\equiv \Gamma^{S}} v_{A}^{\frac{1-\alpha}{2 \alpha}} v_{B}^{\frac{1-\alpha}{2 \alpha}} .
$$

The principal chooses $\left\{v_{A}, v_{B}\right\}$ to maximize her net expected payoff, $\mathbb{E} W=\left(V-v_{A}-\right.$ $\left.v_{B}\right) P^{S}=\left(V-v_{A}-v_{B}\right) \Gamma^{S} v_{A}^{\frac{1-\alpha}{2 \alpha}} v_{B}^{\frac{1-\alpha}{2 \alpha}}$. The first-order conditions are:

$$
\frac{\partial \mathbb{E} W}{\partial v_{i}}=\Gamma^{S} v_{i}^{\frac{1-\alpha}{2 \alpha}} v_{j}^{\frac{1-\alpha}{2 \alpha}}\left[\left(\frac{1-\alpha}{2 \alpha}\right)\left(\frac{V-v_{i}-v_{j}}{v_{i}}\right)-1\right]=0, \quad i, j \in\{A, B\}, i \neq j,
$$

which together imply $v_{A}=v_{B}=v\left(\because \Gamma^{S} v_{A}^{\frac{1-\alpha}{2 \alpha}} v_{B}^{\frac{1-\alpha}{2 \alpha}} \neq 0\right)$. Hence, the above conditions imply

$$
v=\frac{1-\alpha}{2} V, \quad \text { and } \quad W=\alpha V .
$$

This, in turn, implies (using (2)):

$$
\frac{e_{A}^{S}}{e_{B}^{S}}=\left(\frac{\eta_{B}-b}{\eta_{B}}\right)^{\frac{\alpha}{1+\alpha}}<1 \Rightarrow e_{A}^{S}<e_{B}^{S} .
$$

We summarize the above results as follows:

Proposition 1 (Simultaneous Efforts). Under simultaneous effort contribution,

- The equilibrium effort levels are given by (2) and the stereotype about B's ability lowers both agents' efforts compared to the situation in which there is no stereotype.

- The stereotype lowers the probability of the project's success.

- As the stereotype becomes more pronounced, i.e., as $b \uparrow$, effort exerted by both agents (hence, the project's chance of success) falls.

- Despite the stereotype, it is optimal for the principal to offer symmetric incentives to both agents: each is paid $v=\left(\frac{1-\alpha}{2}\right) V$, and the principal retains $W=\alpha V$, if the project succeeds.

- The stereotyped agent exerts higher effort although both agents are paid symmetrically.

The stereotype lowers $A$ 's expectation about $B$ 's effort - this lowers $A$ 's effort. $B$ internalizes this and exerts low effort. Although both agents receive symmetric incentives, the stereotyped agent exerts higher effort as her expectation about the marginal productivity of her effort is not biased downward. Thus, the implicit return per unit of effort is lower for the disadvantaged/stereotyped agent. 


\section{Sequential Effort Contribution}

Consider how the principal can sequence effort contributions to mitigate the stereotype's impact. The timing is as follows. The principal offers $\left\{v_{A}, v_{B}\right\}$. Agent $i$ exerts effort; after observing $i$ 's effort, $j$ exerts effort, $i, j \in\{A, B\}, i \neq j$. The project concludes and payments are made.

Advantaged Agent is the Leader. The principal assigns agent $A$ as leader in effort contribution. Given $v_{A}, v_{B}$ and $e_{A}$, the follower, $B$, solves

$$
\max _{e_{B} \geq 0} \mathbb{E} U_{B}=v_{B} \eta_{A}^{\alpha} \eta_{B}^{\alpha} e_{A}^{1-\alpha} e_{B}^{1-\alpha}-\frac{1}{2} e_{B}^{2}
$$

which gives the follower's best-response function (the superscript $F$ denotes the follower):

$$
e_{B}^{F}=\left[(1-\alpha) v_{B} \eta_{A}^{\alpha} \eta_{B}^{\alpha}\right]^{\frac{1}{1+\alpha}} e_{A}^{\frac{1-\alpha}{1+\alpha}}
$$

The leader, $A$, incorporates the follower's best response in her effort choice problem:

$$
\max _{e_{A} \geq 0} \mathbb{E} U_{A}=v_{A} \underbrace{\left[\eta_{A}^{\alpha}\left(\eta_{B}-b\right)^{\alpha} e_{A}^{1-\alpha}\left(\hat{e}_{B}^{F}\right)^{1-\alpha}\right]}_{\mathbb{E}_{A}(P)}-\frac{1}{2} e_{A}^{2}=v_{A} v_{B}^{\frac{1-\alpha}{1+\alpha}}(1-\alpha)^{\frac{1-\alpha}{1+\alpha}} \eta_{A}^{\frac{2 \alpha}{1+\alpha}}\left(\eta_{B}-b\right)^{\frac{2 \alpha}{1+\alpha}} e_{A}^{\frac{2(1-\alpha)}{1+\alpha}}-\frac{1}{2} e_{A}^{2} .
$$

Note that $A$ underestimates not only the marginal productivity of $B$ 's effort, but also the latter's effort due to the stereotype. When incorporating the follower's effort into her optimization problem, $A$ uses $\hat{e}_{B}^{F}=\left[(1-\alpha) v_{B} \eta_{A}^{\alpha}\left(\eta_{B}-b\right)^{\alpha}\right]^{\frac{1}{1+\alpha}} e_{A}^{\frac{1-\alpha}{1+\alpha}}$ rather than B's actual effort, $e_{B}^{F}$, given by (4). The optimal effort of the leader, $A$, is:

$$
e_{A}^{L}=\left(\frac{2}{1+\alpha}\right)^{\frac{1+\alpha}{4 \alpha}}(1-\alpha)^{\frac{1}{2 \alpha}} v_{A}^{\frac{1+\alpha}{4 \alpha}} v_{B}^{\frac{1-\alpha}{4 \alpha}} \eta_{A}^{\frac{1}{2}}\left(\eta_{B}-b\right)^{\frac{1}{2}}
$$

where the superscript $L$ denotes that $A$ is the leader. Using (4) and (5), we have:

$$
e_{B}^{F}=\left(\frac{2}{1+\alpha}\right)^{\frac{1-\alpha}{4 \alpha}}(1-\alpha)^{\frac{1}{2 \alpha}} v_{A}^{\frac{1-\alpha}{4 \alpha}} v_{B}^{\frac{1+\alpha}{4 \alpha}} \eta_{A}^{\frac{1}{2}} \eta_{B}^{\frac{\alpha}{1+\alpha}}\left(\eta_{B}-b\right)^{\frac{1-\alpha}{2(1+\alpha)}}
$$

It is easy to see that $\frac{\partial e_{A}^{L}}{\partial b}<0$ and $\frac{\partial e_{B}^{F}}{\partial b}<0$, i.e., as $b \uparrow$, the effort of both agents decreases.

The probability of the project's success is (using (5) and (6)):

$$
P^{A}=\eta_{A}^{\alpha} \eta_{B}^{\alpha}\left(e_{A}^{L}\right)^{1-\alpha}\left(e_{B}^{F}\right)^{1-\alpha}=\underbrace{\left(\frac{2}{1+\alpha}\right)^{\frac{1-\alpha}{2 \alpha}}(1-\alpha)^{\frac{1-\alpha}{\alpha}} \eta_{A} \eta_{B}^{\frac{2 \alpha}{1+\alpha}}\left(\eta_{B}-b\right)^{\frac{1-\alpha}{1+\alpha}}}_{\equiv \Gamma^{A}} v_{A}^{\frac{1-\alpha}{2 \alpha}} v_{B}^{\frac{1-\alpha}{2 \alpha}} .
$$

The principal chooses $\left\{v_{A}, v_{B}\right\}$ to maximize her net expected payoff, $\mathbb{E} W=\left(V-v_{A}-\right.$ $\left.v_{B}\right) P^{A}=\left(V-v_{A}-v_{B}\right) \Gamma^{A} v_{A}^{\frac{1-\alpha}{2 \alpha}} v_{B}^{\frac{1-\alpha}{2 \alpha}}$. The first-order conditions are:

$$
\frac{\partial \mathbb{E} W}{\partial v_{i}}=\Gamma^{A} v_{i}^{\frac{1-\alpha}{2 \alpha}} v_{j}^{\frac{1-\alpha}{2 \alpha}}\left[\left(\frac{1-\alpha}{2 \alpha}\right)\left(\frac{V-v_{i}-v_{j}}{v_{i}}\right)-1\right]=0, \quad i, j \in\{A, B\}, i \neq j,
$$


which together imply $\left(\right.$ since $\left.\Gamma^{A} v_{A}^{\frac{1-\alpha}{2 \alpha}} v_{B}^{\frac{1-\alpha}{2 \alpha}} \neq 0\right) v_{A}=v_{B}=v$. Hence,

$$
v=\frac{1-\alpha}{2} V, \quad \text { and } \quad W=\alpha V \text {. }
$$

Thus, not only are the payments to agents symmetric across the leader and follower, they are also the same as those in the simultaneous efforts benchmark. This implies (using (5) and (6)):

$$
\frac{e_{A}^{L}}{e_{B}^{F}}=\underbrace{\left(\frac{2}{1+\alpha}\right)^{\frac{1}{2}}}_{>1} \underbrace{\left(\frac{\eta_{B}-b}{\eta_{B}}\right)^{\frac{\alpha}{1+\alpha}}}_{<1} .
$$

Hence, if the stereotype is sufficiently strong ( $b$ high), even as leader, $A$ exerts lower effort.

Comparing (2), (5) and (6) we get (since the $v_{i}$ 's, $i \in\{A, B\}$, are the same in the two cases):

$$
\frac{e_{A}^{L}}{e_{A}^{S}}=\left(\frac{2}{1+\alpha}\right)^{\frac{1+\alpha}{4 \alpha}}>1, \quad \text { and } \quad \frac{e_{B}^{F}}{e_{B}^{S}}=\left(\frac{2}{1+\alpha}\right)^{\frac{1-\alpha}{4 \alpha}}>1
$$

i.e., both agents exert higher effort with $A$ as leader than under simultaneous moves; hence, $P^{A}>P^{S}$. Even with a strong stereotype (high $b$ ), assigning $A$ as leader improves the chances of the project's success. The principal's expected payoff is, thus, higher with $A$ as leader since the payments in case of success are the same.

We summarize the above results in the following proposition:

Proposition 2 (Advantaged Agent as Leader). Suppose the principal assigns agent A, who believes the negative stereotype about B's ability, as the leader in effort contribution. Then,

- The efforts of the leader (A) and follower (B) are given by (5) and (6), respectively; both agents exert higher effort relative to simultaneous effort contributions.

- The project's success chance is always higher when $A$ is the leader as compared to the simultaneous effort contribution game.

- As the stereotype about $B$ becomes stronger, i.e., as $b \uparrow$, the effort of both agents falls.

- Despite the stereotype and sequential efforts, the principal offers symmetric incentives to both leader and follower, $v=\left(\frac{1-\alpha}{2}\right) V$, and retains $W=\alpha V$, when the project succeeds the same as that under simultaneous efforts.

- The principal is better off under A's leadership than under simultaneous efforts.

- If the stereotype is sufficiently strong, the leader A's effort is lower than the follower B's.

The leader exerts higher effort as she internalizes the effect of her effort choice on the follower's effort decision. The stereotype about the follower's ability (hence, effort choice) lowers $A$ 's effort; this also reduces the follower's effort. However, the first effect dominates and both agents exert higher effort relative to simultaneous effort contributions. Despite being paid symmetrically, agents exert differential efforts due to the stereotype and sequential moves. 
Disadvantaged/Stereotyped Agent is the Leader. Agent $B$ is assigned the leader in effort contribution. Given $v_{A}, v_{B}$ and $e_{B}, A$ 's (the follower's) problem is

$$
\max _{e_{A} \geq 0} \mathbb{E} U_{A}=v_{A} \underbrace{\left[\eta_{A}^{\alpha}\left(\eta_{B}-b\right)^{\alpha} e_{A}^{1-\alpha} e_{B}^{1-\alpha}\right]}_{\mathbb{E}_{A}(P)}-\frac{1}{2} e_{A}^{2},
$$

Note that, that even after observing B's effort, $A$ underestimates the chances of the project's success due to the stereotype. However, in contrast to earlier cases, $A$ does not underestimate $B$ 's effort, which she observes before choosing $e_{A}$. The follower's effort is determined by her best-response function (the superscript $F$ denotes that $A$ is the follower):

$$
e_{A}^{F}=\left[(1-\alpha) v_{A} \eta_{A}^{\alpha}\left(\eta_{B}-b\right)^{\alpha}\right]^{\frac{1}{1+\alpha}} e_{B}^{\frac{1-\alpha}{1+\alpha}}
$$

$B$, as leader, incorporates (8) in her effort choice problem:

$\max _{e_{B} \geq 0} \mathbb{E} U_{B}=v_{B} \underbrace{\left[\eta_{A}^{\alpha} \eta_{B}^{\alpha}\left(\hat{e}_{A}^{F}\right)^{1-\alpha} e_{B}^{1-\alpha}\right]}_{\mathbb{E}_{B}(P)}-\frac{1}{2} e_{B}^{2}=v_{B} v_{A}^{\frac{1-\alpha}{1+\alpha}}(1-\alpha)^{\frac{1-\alpha}{1+\alpha}} \eta_{A}^{\frac{2 \alpha}{1+\alpha}} \eta_{B}^{\alpha}\left(\eta_{B}-b\right)^{\frac{\alpha(1-\alpha)}{1+\alpha}} e_{B}^{\frac{2(1-\alpha)}{1+\alpha}}-\frac{1}{2} e_{B}^{2}$,

and her optimal effort is:

$$
e_{B}^{L}=\left(\frac{2}{1+\alpha}\right)^{\frac{1+\alpha}{4 \alpha}}(1-\alpha)^{\frac{1}{2 \alpha}} v_{A}^{\frac{1-\alpha}{4 \alpha}} v_{B}^{\frac{1+\alpha}{4 \alpha}} \eta_{A}^{\frac{1}{2}} \eta_{B}^{\frac{1+\alpha}{4}}\left(\eta_{B}-b\right)^{\frac{1-\alpha}{4}}
$$

where the superscript $L$ denotes that $B$ is the leader. Further, using (8) and (9), we have:

$$
e_{A}^{F}=\left(\frac{2}{1+\alpha}\right)^{\frac{1-\alpha}{4 \alpha}}(1-\alpha)^{\frac{1}{2 \alpha}} v_{A}^{\frac{1+\alpha}{4 \alpha}} v_{B}^{\frac{1-\alpha}{4 \alpha}} \eta_{A}^{\frac{1}{2}} \eta_{B}^{\frac{1-\alpha}{4}}\left(\eta_{B}-b\right)^{\frac{1+\alpha}{4}}
$$

It is easy to see that $\frac{\partial e_{B}^{L}}{\partial b}<0$ and $\frac{\partial e_{A}^{F}}{\partial b}<0$, i.e., as $b \uparrow$, the effort of both agents decreases.

The probability of the project's success is (using (9) and (10)):

$$
P^{B}=\eta_{A}^{\alpha} \eta_{B}^{\alpha}\left(e_{A}^{F}\right)^{1-\alpha}\left(e_{B}^{L}\right)^{1-\alpha}=\underbrace{\left(\frac{2}{1+\alpha}\right)^{\frac{1-\alpha}{2 \alpha}}(1-\alpha)^{\frac{1-\alpha}{\alpha}} \eta_{A} \eta_{B}^{\frac{1+\alpha}{2}}\left(\eta_{B}-b\right)^{\frac{1-\alpha}{2}}}_{\equiv \Gamma^{B}} v_{A}^{\frac{1-\alpha}{2 \alpha}} v_{B}^{\frac{1-\alpha}{2 \alpha}}
$$

The principal chooses $\left\{v_{A}, v_{B}\right\}$ to maximize her net expected payoff, $\mathbb{E} W=\left(V-v_{A}-\right.$ $\left.v_{B}\right) P^{B}=\left(V-v_{A}-v_{B}\right) \Gamma^{B} v_{A}^{\frac{1-\alpha}{2 \alpha}} v_{B}^{\frac{1-\alpha}{2 \alpha}}$. The first-order conditions are:

$$
\frac{\partial \mathbb{E} W}{\partial v_{i}}=\Gamma^{B} v_{i}^{\frac{1-\alpha}{2 \alpha}} v_{j}^{\frac{1-\alpha}{2 \alpha}}\left[\left(\frac{1-\alpha}{2 \alpha}\right)\left(\frac{V-v_{i}-v_{j}}{v_{i}}\right)-1\right]=0, \quad i, j \in\{A, B\}, i \neq j,
$$

which together imply $v_{A}=v_{B}=v\left(\right.$ since $\left.\Gamma^{B} v_{A}^{\frac{1-\alpha}{2 \alpha}} v_{B}^{\frac{1-\alpha}{2 \alpha}} \neq 0\right)$. Hence,

$$
v=\frac{1-\alpha}{2} V, \quad \text { and } \quad W=\alpha V .
$$

Again, the payments to agents are symmetric, and the same as those under simultaneous efforts. 
This, along with (9) and (10), implies:

$$
\frac{e_{A}^{F}}{e_{B}^{L}}=\left(\frac{1+\alpha}{2}\right)^{\frac{1}{2}}\left(\frac{\eta_{B}-b}{\eta_{B}}\right)^{\frac{\alpha}{2}}<1
$$

The stereotyped agent, as leader, always exerts higher effort than the follower. As the stereotype against $B$ 's ability becomes stronger, her relative effort increases. Hence, the implicit return per unit of effort is lower for the stereotyped agent.

Comparing (2), (9) and(10) we get (since the $v_{i}$ 's, $i \in\{A, B\}$, are the same in the two cases):

$$
\frac{e_{A}^{F}}{e_{A}^{S}}=\left(\frac{2}{1+\alpha}\right)^{\frac{1-\alpha}{4 \alpha}}\left(\frac{\eta_{B}}{\eta_{B}-b}\right)^{\frac{1-\alpha}{4}}>1, \quad \text { and } \quad \frac{e_{B}^{L}}{e_{B}^{S}}=\left(\frac{2}{1+\alpha}\right)^{\frac{1+\alpha}{4 \alpha}}\left(\frac{\eta_{B}}{\eta_{B}-b}\right)^{\frac{(1-\alpha)^{2}}{4(1+\alpha)}}>1
$$

Hence, both agents always exert higher effort when the stereotyped agent leads as compared to simultaneous effort contribution. Thus, $P^{B}>P^{S}$. As the stereotype becomes stronger $(b \uparrow)$, efforts under $B$ 's leadership increase relative to simultaneous moves.

We summarize the above results in the following proposition:

Proposition 3 (Disadvantaged/Stereotyped Agent as Leader). Suppose the principal assigns the stereotyped agent, $B$, as leader in effort contribution. Then,

- The efforts of the leader (B) and follower (A) are given by (9) and (10), respectively; both agents exert higher effort relative to simultaneous effort contributions.

- The project's success chance is always higher with the stereotyped agent, B, as leader as compared to simultaneous effort contributions.

- As the stereotype about $B$ becomes stronger, i.e., as $b \uparrow$, the effort of both agents falls.

- Despite the stereotype and sequential effort contributions, the principal offers symmetric incentives to both agents, $v=\left(\frac{1-\alpha}{2}\right) V$, and retains $W=\alpha V$, when the project succeedsthe same as that under simultaneous efforts and with $A$ as leader.

- The principal is better off under B's leadership than under simultaneous efforts.

- The disadvantaged agent, B, always exerts higher effort than the advantaged agent, $A$.

Agent $B$, as leader, exerts high effort since she internalizes the impact of her choice on the follower's effort and, unlike $A$, she does not believe the negative stereotype. This mitigates $A$ 's low expectation about $B$ 's effort as $A$ no longer has to rely on her (biased) belief about $B$ 's effort. This improved expectation about the project's success increases the marginal productivity of $A$ 's effort and prompts $A$ to contribute higher effort. Hence, having $B$ (who is stereotyped) as leader in effort contribution always improves both agents' efforts. The stereotype and order of moves imply that the return per unit of effort is lower for the disadvantaged agent relative to the advantaged agent.

Which Sequential Contribution Regime is Better? Propositions 2 and 3 show that with either $A$ or $B$ as leader, both agents exert higher effort relative to simultaneous effort 
contribution. Consider the project's success probability under the two regimes, (7) and (11):

$$
\frac{P^{B}}{P^{A}}=\left(\frac{\eta_{B}}{\eta_{B}-b}\right)^{\frac{(1-\alpha)^{2}}{2(1+\alpha)}}>1 .
$$

This, combined with the earlier result that $P^{A}>P^{S}$, implies $P^{B}>P^{A}>P^{S}$. Since the principal's share is the same under all regimes, the principal's expected payoff is the highest with $B$ as leader and the lowest under simultaneous efforts. Furthermore, (12) implies that, as the stereotype becomes stronger, the gain from $B$ 's leadership increases. If there is no stereotype $(b=0)$, both leadership regimes result in the same outcome.

We summarize the above results in the following proposition:

Proposition 4 (Comparison of Sequential Effort Contribution Outcomes). Comparing the leadership regimes, we have:

- The chance of the project's success and the principal's expected surplus are highest with the stereotyped agent, B, as leader and the lowest when effort contribution is simultaneous.

- As the stereotype becomes stronger ( $b \uparrow)$, the gain from $B$ 's leadership relative to A's leadership increases.

The leader always internalizes the effect of her effort contribution on the follower's effort choice. However, if the stereotype is extreme ( $b$ is high), placing $A$ as leader does not increase her effort as much since she believes that $B$ 's marginal productivity of effort is much lower than it actually is and underestimates $B$ 's effort. This problem is mitigated if the stereotyped agent is assigned leader in effort contribution; $B$ 's high effort increases the marginal productivity of $A$ 's effort (despite the stereotype), so she also exerts higher effort.

\section{Discussion}

We use a simple model to focus our analysis on the effect of background-based stereotypes on efforts in a team setting with peer effects. Placing stereotyped agents as leaders in effort contribution mitigates the effect of stereotypes and increases the chances of the project's success. Although both agents are offered symmetric incentives, which may seem non-discriminatory, the disadvantaged/stereotyped agent exerts higher effort under the principal's regime of choice. Thus, the disadvantaged agent bears the cost of the stereotype through a lower implicit return per unit of effort. Our analysis would be applicable to relatively short time frames compared to the time it takes to change negative stereotypes. Future work could incorporate the change in stereotypes over time as individuals from different social backgrounds interact repeatedly. 


\section{References}

[1] Andreoni, J. (2006). Leadership Giving in Charitable Fund-Raising. Journal of Public Economic Theory, 8, 1-22.

[2] Bag, P.K. and Roy, S. (2011). On Sequential and Simultaneous Contributions under Incomplete Information. International Journal of Game Theory, 40, 119-145.

[3] Bag, P.K., Saha, B. and Sikdar, S. (2015). Bias, Prejudice and Identity Neutral Policy. Mimeo.

[4] Banerjee, A., Bertrand, M., Datta, S. and Mullainathan, S. (2009). Labor Market Discrimination in Delhi: Evidence from a Field Experiment. Journal of Comparative Economics, $37,14-27$.

[5] Bertrand, M. and Mullainathan, S. (2004). Are Emily and Greg More Employable than Lakisha and Jamal? A Field Experiment on Labor Market Discrimination. American Economic Review, 94, 991-1013.

[6] Chaudhuri, S. and Sethi, R. (2008). Statistical Discrimination with Peer Effects: Can Integration Eliminate Negative Stereotypes. Review of Economic Studies, 75, 579-596.

[7] Coate, S. and Loury, G.C. (1993). Will Affirmative Action Policies Eliminate Negative Stereotypes? American Economic Review, 83, 1220-1240.

[8] Compte, O. and Postlewaite, A. (2004). Confidence-Enhanced Performance. American Economic Review, 94, 1536-1557.

[9] Gervais, S. and Goldstein, I. (2007). The Positive Effects of Biased Self-Perceptions in Firms. Review of Finance, 11, 453496.

[10] Hermalin, B. (1998). Toward an Economic Theory of Leadership: Leading by Example. American Economic Review, 88, 1188-1206.

[11] Hoff, K. and Stiglitz, J.E. (2010). Equilibrium Fictions: A Cognitive Approach to Societal Rigidity. American Economic Review, 100, 141-146.

[12] Holmström, B. (1982). Moral Hazard in Teams. Bell Journal of Economics, 13, 324340.

[13] Jones, M. (2002). Social Psychology of Prejudice, Saddle River, NJ: Pearson Education.

[14] Kochar, A. (2010). Affirmative Action through Quotas: The Effect on Learning In India. Mimeo.

[15] Lazear, E.P. and Shaw, K.L. (2007). Personnel Economics: The Economist's View of Human Resources. Journal of Economic Perspectives, 21, 91-114.

[16] Sekhri, S. (2012). Does Academic Peer Quality Promote Solidarity? Evidence from Caste Based Peer Effects in India. Mimeo.

[17] Shaw, D.G. and Schneier, C.E. (1995). Team Measurements and Rewards: How Some Companies are Getting It Right. Human Resource Planning, 18, 34-49.

[18] Varian, H.R. (1994). Sequential Contributions to Public Goods. Journal of Public Economics, 53, 165-186. 